\title{
Liposome formation with wool lipid extracts rich in ceramides
}

\author{
R. Ramírez ${ }^{1}$, M. Martí ${ }^{1}$, A. Cavaco-Paulo², R. Silva ${ }^{2}$, A. de la Maza ${ }^{1}$, J.L. Parra ${ }^{1}$, and L. Coderch ${ }^{1}$ \\ ${ }^{1}$ IQAC (CSIC), Jordi Girona 18-26, 08034 Barcelona, Spain, and ${ }^{2}$ Universidade do Minho, Campus de Azurém, \\ Guimarães, Portugal
}

\begin{abstract}
Internal wool lipids (IWLs) are rich in cholesterol, free fatty acids, cholesteryl sulfate, and, mainly, ceramides. The repairing effect of these lipids structured as liposomes was demonstrated by reinforcing the skin-barrier integrity and increasing the water-holding capacity when applied onto the skin. This work was focused on the formation of liposomes with IWLs rich in ceramides, obtained at pilot plant level with organic solvent extraction by using methanol and acetone. The lipid composition of the two extracts was quantitatively analyzed. IWL extracts containing different amounts of sterol sulfate were used to form liposomes at physiologic $\mathrm{pH}$. Vesicle size distribution, polydispersity index, and zeta potential of all liposomes were determined to characterize them and to study their stability. The results obtained showed that IWL extract composition, which was different depending on the extraction methodologies used, greatly influences the characteristics of the liposomes formed. Vesicular size and polydispersity index liposomes were smaller when the extract composition contained a higher proportion of either free fatty acids or sterol sulfate. Moreover, liposome stability was improved when some amount of sterol sulfate was added to the composition of methanol and acetone extracts. This natural mixture with keratinaceous origin could have a special interest for cosmetic or dermopharmaceutical companies.
\end{abstract}

\section{Introduction}

Wool is a natural fiber predominantly composed of keratin proteins with an external lipid content (lanolin) and with a minor internal lipid content (1.5\%). Internal wool lipids (IWLs) are rich in cholesterol, free fatty acids, cholesteryl sulfate, and, mainly, ceramides. These lipids resemble those found in membranes of other keratin tissues, such as human hair or stratum corneum from skin (Coderch et al., 1995; Schaefer and Redelmeier, 1996).

Ceramides, which are derivatives of sphingosine or phytosphingosine in amide linkage with nonhydroxy, $\alpha$-hydroxy, and $\omega$-hydroxy acids, are the main component of the intercellular lipids in stratum corneum. These lipids play an essential role in maintaining and structuring the lipid barrier, which affords protection against external insults and water loss through the skin (Coderch et al., 2003b; Wartewig and Neubert, 2007).

The presence of phospholipids was considered a condition for the formation of bilayers and liposomes, since bilayer-forming lipids consist predominantly of phospholipids in all other biological membranes. However, stratum corneum lipids, in which phospholipids are practically absent, have been proven to form stable liposomes (Gray and White, 1979).

Liposomes represent a model for more complex biological membranes in biophysical and medical research because of the lipid bilayer structural similarity. Moreover, liposomes have been the subject of numerous studies because of their importance as microencapsulators for drug delivery and their applications in cosmetics (Teschke and de Souza, 2002; Lian and Ho, 2001; Betz et al., 2005). Liposomes containing synthetic lipid mixtures approximating the composition of stratum corneum lipids have been tested as a model in order to study stratum corneum properties and as a formulation to improve skin condition or to repair skin damage (de la Maza et al., 1995; Coderch et al., 2002a).

Internal lipids of keratin fibers, such as wool and human hair, extracted at laboratory level have been shown to form stable liposomes and hence bilayer 
structures in aqueous media (Körner et al., 1995; Fonollosa et al., 2000b, 2004). Besides, IWL liposome application has been proved to confer better properties to human hair and skin. Whereas IWL liposomes applied onto the skin reinforce the skin-barrier integrity and increase its water-holding capacity (Corderch et al., 2002a; de Pera et al., 2000), the application of IWL liposomes to chemically treated hair samples leads to an improvement in the mechanical strength of the fibers (Méndez et al., 2008). Therefore, IWL extracts structured as liposomes seem suitable for incorporation into pharmaceutical or cosmetics formulations in the treatment and care of skin (Corderch et al., 2000) and human hair (Kelly et al., 2007).

This work was focused on the formation of liposomes with IWLs rich in ceramides, obtained at pilot plant level with nonchlorinated organic solvent extraction, using methanol and acetone as solvents. The characteristics of the liposomes formed with the two IWL extracts, whose composition is different, were studied and improved by adding different amounts of sterol sulfate.

\section{Experimental procedures}

\section{Extraction procedures}

Raw Spanish Merino wool samples supplied by SAIPEL (Terrassa, Spain) were used for lipid extraction. Prior to the extraction, raw wool was industrially cleaned, following the procedure previously described (Ramírez et al., 2008). IWLs were obtained at pilot plant level with organic solvent extraction, using methanol and acetone. The extraction procedure consisted in a pump-forced reflow system. First, $28 \mathrm{~kg}$ of wool were extracted for 7.5 hours. The extraction temperature was $45^{\circ} \mathrm{C}$ for methanol and $40^{\circ} \mathrm{C}$ for acetone. After distillation, the two lipid extracts were concentrated and stored in chloroform/ methanol $(2 / 1, \mathrm{v} / \mathrm{v})$ at $-20^{\circ} \mathrm{C}$ until their analysis (Ramírez et al., 2008).

\section{Lipid analysis}

The quantitative analysis of the IWLs was performed by thin-layer chromatography coupled to an automated flame ionization detector (TLC-FID) Iatroscan MK-5 analyzer (Iatron, Tokyo, Japan), following the analysis methodology referred to in earlier works (Fonollosa et al., 2000a; Corderch et al., 2002b). Samples (15-20 $\mu \mathrm{g}$ ) were spotted on silica gel S-III chromarods, using an SES (Nieder-Olm, Germany) 3202/15-01 sample spotter.

An analysis of apolar and polar compounds was performed by developing the rods initially to a distance of 10 cm with n-hexane/diethyl ether/formic acid (53:17:0.3, by volume) to separate apolar and polar lipids. After a partial scan of $85 \%$ to quantify and eliminate the apolar lipids, a second development, again to a distance of 10 $\mathrm{cm}$, was performed with chloroform/n-hexane/methanol/acetone (55:5:3:7, by volume) to separate the ceramides. Following a partial scan of $85 \%$ to quantify and eliminate the ceramides, a third development, again to a distance of $10 \mathrm{~cm}$, was performed with chloroform/ methanol/formic acid (57:12:0.3, by volume) to separate and quantify, after a total scan of $100 \%$, the glycosilceramides and sterol sulfate. After each elution, the rods were heated for 5 minutes at $60^{\circ} \mathrm{C}$ to dry the remaining solvent. The experimental conditions were: air flow $2000 \mathrm{~mL} / \mathrm{min}$, hydrogen flow 160-180 mL/min, and scanning speed 2-3 $\mathrm{mm} / \mathrm{s}$. Data were processed with Boreal version 2.5 software (JMBS Development, Grenoble, France).

These procedures were applied to the following standard compounds: palmitic acid and cholesterol from Fluka Chemicals (Buchs, Switzerland), type II ceramides, cholesterol ester, galactoceramides, and sodium cholesteryl sulfate from Sigma (St. Louis, Missouri, USA) to determine the corresponding calibration curves for quantification of each compound.

\section{Liposome formation and evaluation}

IWLs from methanol extract and IWLs obtained with acetone extraction alone or with some extra amount of sterol sulfate (sodium cholesteryl sulfate) were used to form liposomes. Liposomes were prepared by evaporating the chloroform/methanol solvent to dryness under a stream of dry nitrogen to form a thin film on the flask. The lipid film was hydrated to give a final lipid concentration of $1.5 \mathrm{mg} / \mathrm{mL}$. The buffer used was an $0.2 \mathrm{M}$ phosphate solution with $0.9 \% \mathrm{NaCl}$ adjusted to a $\mathrm{pH}$ of 7.4 .

Liposomes were formed by the application of ultrasounds. The experimental setup used was composed of a probe-type ultrasound source $(20 \mathrm{kHz}$ Sonics and Materials Vibracell CV 33, Newtown, Connecticut, USA) fitted with a $3 \mathrm{~mm}$ diameter titanium microtip. Power delivery was controlled as percentage amplitude. The reaction vessel was an open glass cell (diameter $19 \mathrm{~mm}$ and height $75 \mathrm{~mm}$ ), which contained $15 \mathrm{~mL}$ of sample solution. The sonochemical reactor temperature was controlled via a thermo-stated water bath with a heat exchanger placed within a thermo jacket cell; this gave a steady operating temperature of $50^{\circ} \mathrm{C}\left( \pm 1^{\circ} \mathrm{C}\right)$, which was higher than the transition temperature of IWLs (Corderch et al., 2003a; Méndez et al., 2007). Sonication was carried out with a total treatment of 18 minutes and monitored in 3 minutes increments. All the experiments were made by using a pulsed duty cycle of 8 seconds on, 2 seconds off. The ultrasonic treatment of the liposomes was carried out at an amplitude of $40 \%$ and using the depth of $38.5 \mathrm{~mm}$ (from the base of the vessel). These conditions were selected according to preliminary studies (Little et al., 2007; Silva et al., 2008). 
Liposome vesicle size distribution, polydispersity index, and zeta potential were measured by using dynamic light scattering (Zetasizer Nano ZS, ZEN3600; Malvern Instruments Ltd., Malvern, Worcestershire, UK). Samples were diluted from 1.5 to $0.15 \mathrm{mg} / \mathrm{mL}$. A glass cell was used to determine size distribution and polydispersity index, whereas zeta potential was measured by using folded capillary cells. The cells were filled with the samples, and all the experiments were thermostatically controlled $\left(50^{\circ} \mathrm{C}\right)$. The samples were measured at a scattering angle of 173 degrees. Thus, the data obtained were collected and analyzed by using the program DTS (dispersion technology software) provided by Malvern Instruments Ltd.

\section{Results and discussion}

\section{Lipid extraction and analysis}

Raw Spanish Merino wool was extracted at pilot plant level in order to obtain the lipid extract rich in ceramides. Merino wool from Spain was used because its internal lipid composition resembles that found in the stratum corneum from the skin (Corderch et al., 2002b). The extraction was performed with methanol and acetone, using the conditions described in the experimental procedures section. These solvents were chosen as extractors to avoid the use of chlorinated solvents, which are widely used at laboratory level (Corderch et al., 2002b).

Quantitative analysis of the two different lipid extracts were carried out by TLC-FID, following the methodology reported in earlier papers (Fonollosa et al., 2000a; Corderch et al., 2002b). The different lipid families were quantified as sterol esters (ST-ESs), free fatty acids (FFAs), sterols (STs), ceramides (CERs), glycosilceramides (GLYCERs), and sterol sulfate (ST-SUL). The amount of these components analyzed, expressed in percentages over total weight extracted, is listed in Table 1, and expressed in percentages on total lipid analyzed (\% o.l.a.), as shown in Figure 1.

A comparison of the extracts obtained with solvent extraction at pilot plant level showed that methanol extract was richer in FFAs and polar lipids, such as GLYCERs and ST-SUL, whereas acetone extract was richer in ST-ESs. The percentage of STs and CERs was slightly higher in acetone extract than in methanol extract. The percentage of the main lipids analyzed was similar in both extracts: $65 \%$ for methanol extract and $63 \%$ for acetone extract. The rest of the compounds, which were not quantified, could be triglycerides, fatty alcohols, oxidized compounds, and proteins.

\section{Liposome formation with methanol and acetone extracts}

In previous works, the IWLs extracted with Soxhlet at the laboratory level, whose composition is similar to the one present in the stratum corneum, have been demonstrated to be capable of forming liposomes with a stable bilayer structure (Körner et al., 1995; Fonollosa et al, $2000 \mathrm{~b}, 2004$ ). This vesicular structure, which mimics the organized lipid structures of the stratum corneum, offers a suitable strategy for achieving an accurate vehiculization of a particular compound and for incorporating additional lipid content that may reinforce the barrier function of the skin (Schmid and Korting, 1993; Fresta and Puglisi, 1997; Coderch et al., 1999). In a previous study (Coderch et al., 2002a), the topical application effects of the same lipid mixtures formulated in an oily solvent used as an emollient or in an aqueous saline solution structured in liposomes were investigated. The importance of the bilayer structure of the formulations, which induced the highest hydration properties, should be highlighted. Further, in different studies (Coderch et al., 2002a; de Pera et al., 2000), in which the ability of liposomes to repair human skin previously damaged has been evaluated, slightly better results were obtained with IWL liposomes, which contained a mixture of natural CERs, when compared with stratum corneum liposomes (modeling stratum corneum lipids using a

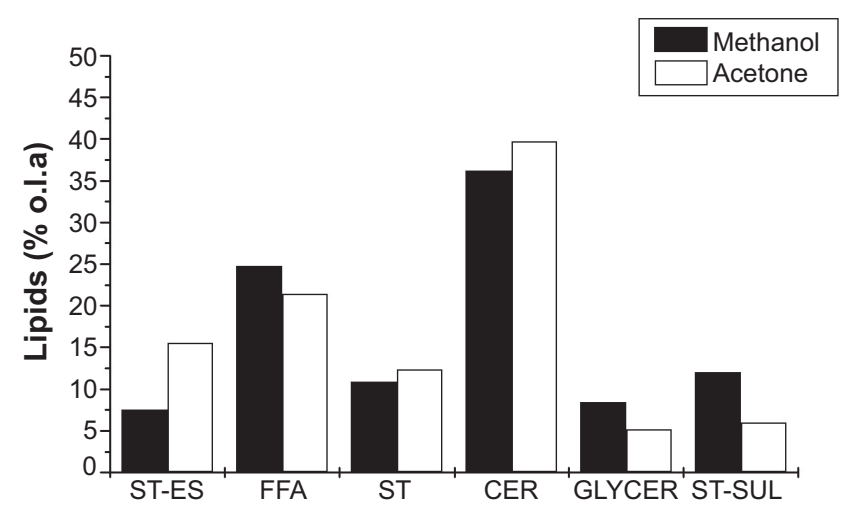

Figure 1. Amount of sterol esters (ST-ESs), free fatty acids (FFAs), sterols (STs), ceramides (CERs), glycosilceramides (GLYCERs), and sterol sulfate (ST-SUL) expressed as percentages of the total lipid weight analyzed (\% o.l.a.).

Table 1. Amount of sterol esters (ST-ESs), free fatty acids (FFAs), sterols (STs), ceramides (CERs), glycosilceramides (GLYCERs), and sterol sulfate (ST-SUL) expressed as percentages of the total lipid weight extracted.

\begin{tabular}{lccccccc}
\hline Extract & ST-ES & FFA & ST & CER & GLYCER & ST-SUL & \% total lipids analyzed \\
\hline Methanol & 4.93 & 16.27 & 7.16 & 23.73 & 5.52 & 7.91 & 65.52 \\
Acetone & 9.75 & 13.50 & 7.75 & 25.00 & 3.25 & 3.75 & 63.00 \\
\hline
\end{tabular}




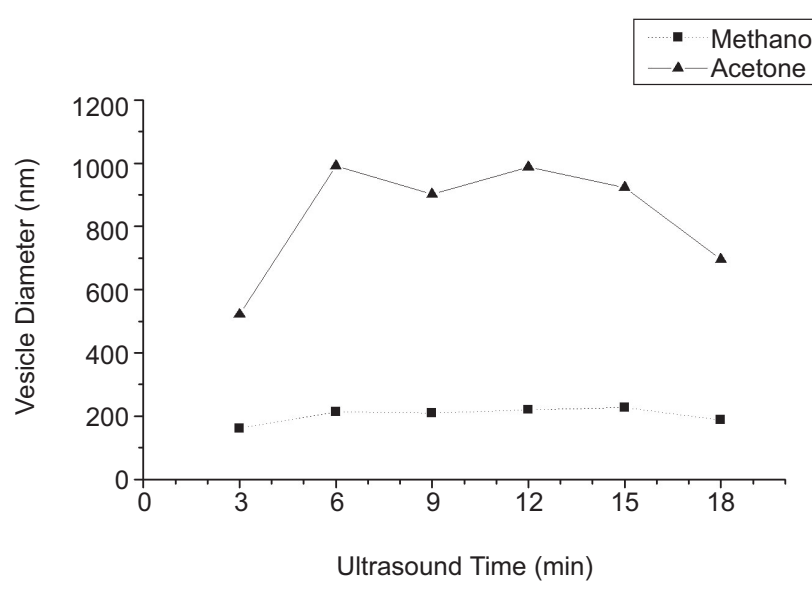

Figure 2. Variation of the vesicle diameter with ultrasound sonication time of IWLs extracted with methanol or acetone.

synthetic lipid mixture), which had only one type of CER present in the formulation.

In the present work, the formation of liposomes was explored by using the different lipid extracts obtained with organic solvent extraction at the pilot plant level, previously analyzed to determine their composition. Liposomes were formed with IWLs extracted by methanol and acetone, using ultrasounds. The ultrasound conditions used, which are detailed in the experimental procedures section, were fixed in all the experiments in order to influence, in the same way, the physical characteristics of the vesicles formed from the different extracts (Pereira-Lachataignerais et al., 2006). Liposomes characteristics were measured with a Zetasizer Nano ZS (Malvern Instruments Ltd., Malvern, Worcestershire, UK) every 3 minutes of sample sonication.

The variation of the vesicle diameter of liposomes prepared from lipids extracted by methanol and acetone with sonication time can be visualized in Figure 2. Higher vesicle diameter was obtained for liposomes formed with IWLs extracted by acetone at all stages of the ultrasound process. Moreover, the polydispersity index after applying ultrasound for 18 minutes was about 0.25 for liposomes formed with methanol extract and 0.50 for liposomes formed with acetone extract. The different behavior between methanol and acetone extracts when liposomes were prepared may be due to the different lipid extract composition.

\section{Addition of sterol sulfate}

The CERs in stratum corneum lipids appear to be insufficient for bilayer formation, perhaps because they are not ionized at physiologic pH. FFAs and ST-SUL, which are also present in stratum corneum lipids, are ionized at physiologic $\mathrm{pH}$ and their presence was reported to be necessary to the bilayer formation (Wertz et al., 1986). Therefore, the different physical characteristics of liposomes formed with acetone extract, with respect to
Table 2. Amount of sterol sulfate (ST-SUL) added and final amount of the different samples.

\begin{tabular}{lcc}
\hline Extract & \% ST-SUL added & \% ST-SUL final \\
\hline \multirow{2}{*}{ Methanol } & 0 & 7.9 \\
& 6 & 13.9 \\
\multirow{3}{*}{ Acetone } & 0 & 3.8 \\
& 3 & 6.8 \\
& 9 & 12.8 \\
\hline
\end{tabular}

The results are expressed as percentages of the total lipid weight extracted.

methanol extract, may be related to the lower amount of FFAs and ST-SUL contained in acetone extract.

In order to improve the characteristics of the liposomes formed, different quantities of ST-SUL were added in acetone and methanol extracts. Whereas methanol extract contained $8 \%$ of ST-SUL in its composition, only $4 \%$ was present in the acetone extract. Therefore, $6 \%$ of ST-SUL was added to methanol extract and $3 \%$ and $9 \%$ of ST-SUL were added to acetone extract to achieve a similar final percentage of ST-SULs in both extracts. Moreover, the composition of the different extracts enriched in ST-SUL approaches to the one present in stratum corneum and hair lipids. The five samples obtained by adding the different amounts of ST-SUL to methanol and acetone extracts are indicated in Table 2.

The formation of liposomes with the five samples, which contain a different amount of ST-SUL, was investigated. These liposomes were formed by the application of ultrasounds for 18 minutes, following the procedure detailed in the experimental section. Liposome vesiclesize distribution, polydispersity index, and zeta potential were measured every 3 minutes of sample sonication.

The results obtained showed that 9 minutes were enough to achieve these vesicles with stable characteristics. The variation of the vesicle diameter, polydispersity index, and zeta potential of these liposomes, with respect to the percentage of ST-SUL, at 9 minutes of sonication, is shown in Figure 3,Figure 4, and Figure 5, respectively.

When the percentage of ST-SUL in acetone extract increases until $7 \%$, the vesicle diameter and the polydispersity index of the liposomes formed diminish abruptly. However, an increase of up to $13 \%$ only slightly diminishes the vesicle diameter and polydispersity index. The physical behavior of liposomes formed by methanol extract is similar when the extracts contained $8 \%$ or $14 \%$ of ST-SUL in their composition. Therefore, liposomes prepared from lipids extracted by acetone could achieve similar vesicular diameter and polydispersity index to liposomes prepared from lipids extracted by methanol when $7 \%$ of ST-SUL is present in their composition.

The results obtained in the zeta potential of the five samples show the different behavior of the liposomes formed by methanol and acetone extracts when the 
Table 3. Stability of liposomes: vesicular diameter (d) and polydispersity index (PI).

\begin{tabular}{|c|c|c|c|c|c|c|c|}
\hline \multirow[b]{2}{*}{ Extract } & \multirow[b]{2}{*}{ \% ST-SUL final } & \multicolumn{2}{|c|}{ Day 0} & \multicolumn{2}{|c|}{ Day 7} & \multicolumn{2}{|c|}{ Day 14} \\
\hline & & $\mathrm{d}(\mathrm{nm})$ & PI & $\mathrm{d}(\mathrm{nm})$ & PI & $\mathrm{d}(\mathrm{nm})$ & $\mathrm{PI}$ \\
\hline \multirow{2}{*}{ Methanol } & 7.9 & 187.5 & 0.247 & 530.6 & 0.393 & 504.6 & 0.269 \\
\hline & 13.9 & 129.7 & 0.159 & 254.3 & 0.351 & 337.7 & 0.409 \\
\hline \multirow{3}{*}{ Acetone } & 3.8 & 695.4 & 0.504 & 485.0 & 0.491 & 354.0 & 0.454 \\
\hline & 6.8 & 269.2 & 0.207 & 327.3 & 0.250 & 353.5 & 0.270 \\
\hline & 12.8 & 125.8 & 0.055 & 158.4 & 0.111 & 160.9 & 0.114 \\
\hline
\end{tabular}

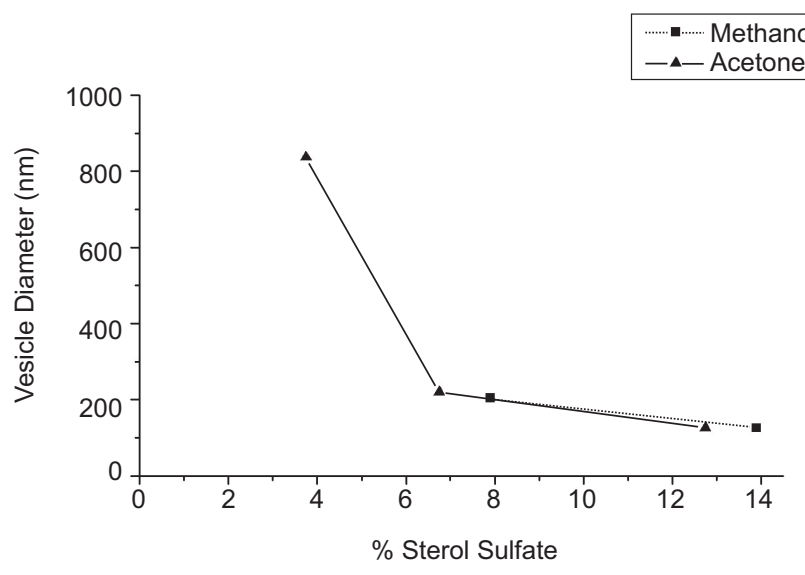

Figure 3. Variation of the vesicle diameter with the percentage of sterol sulfate in the lipid extracts.

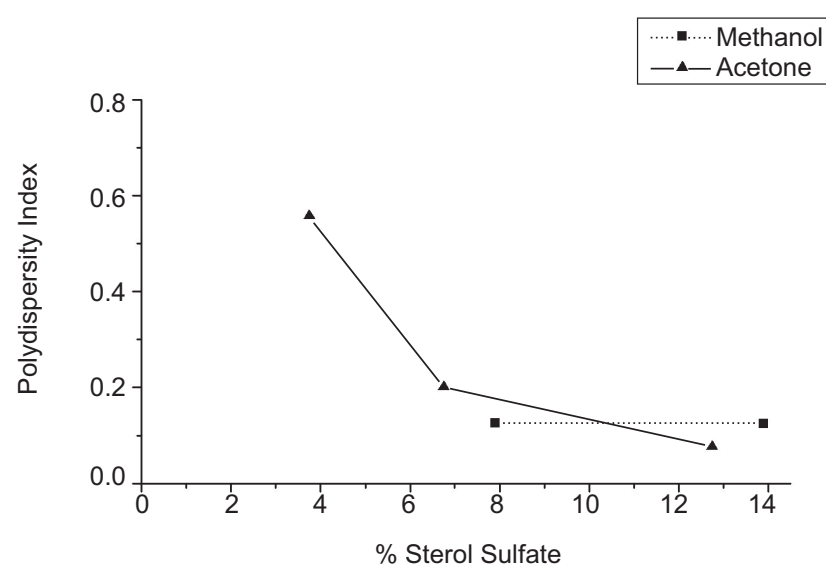

Figure 4. Variation of the polydispersity index with the percentage of sterol sulfate in the lipid extracts.

amount of ST-SUL was modified. Whereas the zeta potential of liposomes formed with acetone extract are almost independent on the percentage of ST-SUL, in liposomes formed with IWLs extracted by methanol, the zeta potential sharply decreases in magnitude when the percentage of ST-SUL increases. The lower value of zeta potential for the methanol extract, compared with the acetone extract, may be due to the higher amount of negative charges because of the higher amount of FFAs and ST-SUL in the methanol extract. However, the zeta potential does not directly depend on the charges of the different components of the lipid bilayer; it is basically related to the

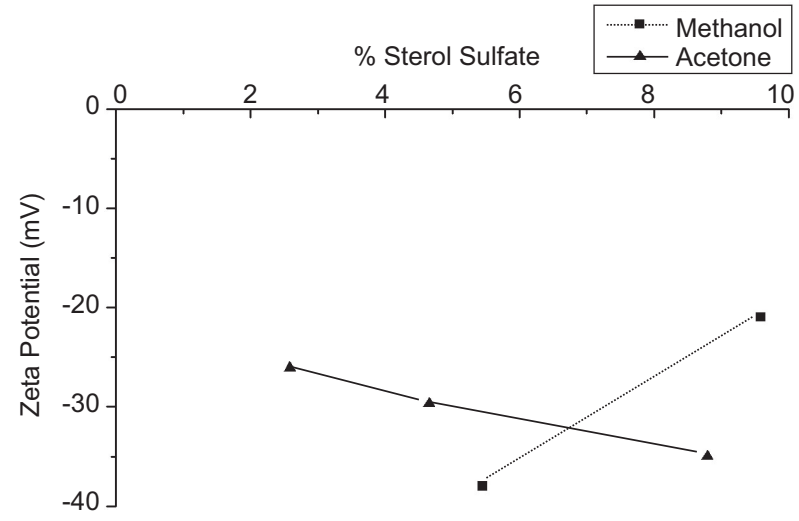

Figure 5. Variation of the zeta potential with the percentage of sterol sulfate in the lipid extracts.

charges on the surface, which depends on the structure of the bilayer and the amount of surface, which is higher when the vesicles are smaller. Therefore, the increase in magnitude for the acetone extract when ST-SUL is added may be mainly due to a decrease in vesicle size, so the negative surface increases. However, when the size is small enough and more ST-SUL is added, the ratio of ST-SUL/FFAs increases and the higher volume of ST-SUL may supplant more molecules of FFAs with the final result of less negative charges at the surface of the vesicles.

\section{Liposomes stability}

In order to study the stability of the methanol and acetone extracts with a different percentage of ST-SUL in their composition, the vesicle diameter and polydispersity index of the different liposome samples were analyzed with a Zetasizer Nano ZS for 14 days. The results obtained are indicated in Table 3 and in Figure 6 and Figure 7.

The results obtained for methanol extract show that the increase of the vesicular diameter is more significant in liposomes prepared with $8 \%$ of ST-SUL (from 187.5 to $530.6 \mathrm{~nm}$ after 7 days since their formation) than in liposomes formed with $14 \%$ of ST-SUL (from 129.7 to $337.7 \mathrm{~nm}$ after 14 days since their formation). Therefore, liposomes prepared from lipids extracted by methanol are more stable during all the study when $14 \%$ of ST-SUL is present in its composition.

The vesicular diameter of the liposomes formed with the original acetone extract with only $4 \%$ of ST-SUL 


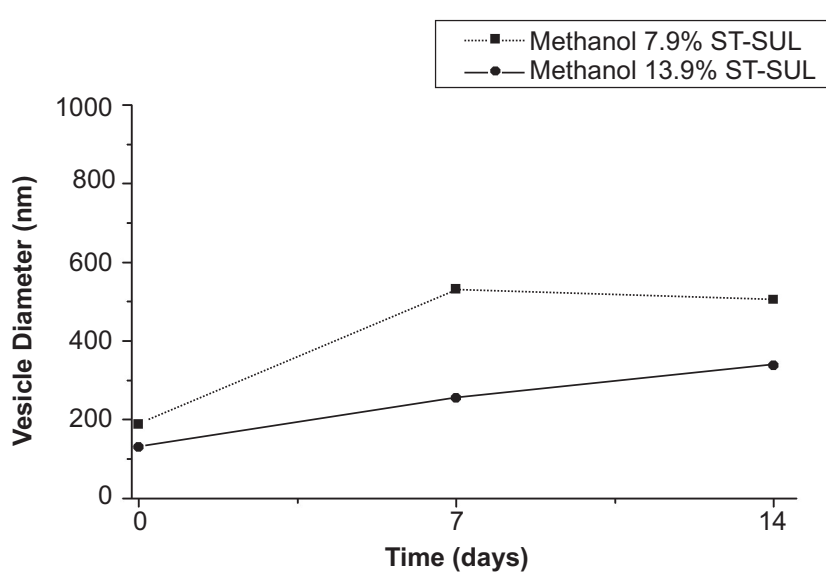

Figure 6. Variation of the vesicle diameter with the time in liposomes formed with methanol adding a different amount of sterol sulfate (ST-SUL).

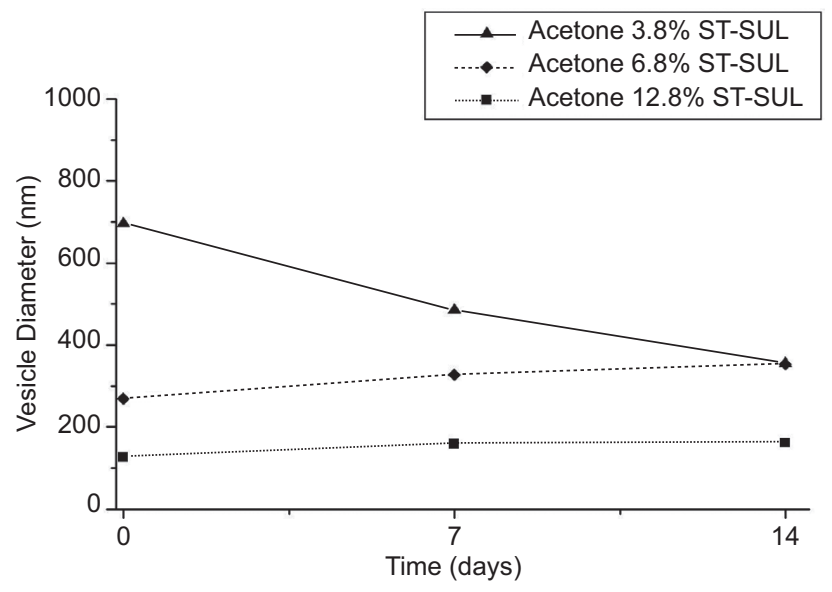

Figure 7. Variation of the vesicle diameter with the time in liposomes formed with acetone adding different amounts of sterol sulphate (ST-SUL).

diminishes after 14 days since their formation. However, the physical characteristics of the liposomes formed with the acetone extract when different percentages of ST-SUL are added in their composition are quite constant during all the study. Consequently, liposomes prepared from lipids extracted by acetone are more stable when 7 and $13 \%$ of ST-SUL is present in its composition.

Even though a longer term study of liposome stability would have been more accurate, the results derived from this 14-day liposome stability study showed that the variation of the vesicular size between the first and the last day diminishes when the percentage of ST-SUL increases in their composition.

Therefore, the addition of ST-SUL in IWL extracts can be used to improve the characteristics and stability of IWL liposomes. Thus, these liposomes enriched in ST-SUL can be applied onto the stratum corneum of skin and hair because of their stable bilayer structure approximating the lipid composition of these keratin tissues. Moreover, these IWL liposome samples could be processed as hydrogels to obtain viscous products suitable for application onto skin and hair (Esposito et al., 2007).

\section{Conclusions}

IWL extract composition is different, depending on the extraction solvent used. IWLs extracted with organic solvents, using methanol, are richer in FFAs and polar lipids (GLYCERs and ST-SUL), whereas IWLs extracted with acetone are richer in apolar lipids, such as ST-ESs.

The IWL extract composition greatly influences the characteristics of the liposomes formed. Vesicular size and polydispersity index of the liposomes formed were smaller when the extract composition contained a high proportion of FFAs or ST-SUL. Moreover, liposomes prepared from lipids extracted by acetone could reach similar vesicular diameter and polydispersity index to liposomes prepared from lipids extracted by methanol when 7\% of ST-SUL is present in their composition.

A clear negative charge is present at the external surface of all liposomes formed with the two extracts. The different behavior of zeta potential and stability by the addition of ST-SUL in liposomes formed by methanol and acetone extracts may be due to the different composition of other lipids in the two extracts.

In the present work, we obtained stable, small liposomes with a low polydispersity index by adding different amounts of ST-SUL to liposomes formed with CERs extracted from wool similar to the present in the stratum corneum and the human hair. This natural mixture, with a keratinaceous origin, could have a special interest for cosmetic or dermopharmaceutical companies.

\section{Acknowledgements}

We acknowledge Mr. G. von Knorring for his expert technical assistance. We are also indebted to the DGICYT Program (PPQ 2002-94136-C02-01 and C02-02) for financial support.

Declaration of interest: The authors report no conflicts of interest. The authors alone are responsible for the content and writing of this paper.

\section{References}

Betz G, Aeppli A, Menshutina N, Leuenberger H (2005). In vivo comparison of various liposome formulations for cosmetic application. Int J Pharm 296, 44-54.

Coderch L, Bondía I, Fonollosa J, Méndez S, Parra JL (2003a). Ceramides from wool: analysis and structere. IFSCC 6, 117-123. 
Coderch L, de Pera M, Fonollosa J, de la Maza A, Parra JL (2002a). Efficacy of stratum corneum lipid supplementation on human skin. Cont Dermat 47, 139-146.

Coderch L, de Pera M, Pérez-Cullell N, Estelrich J, de la Maza A, Parra JL (1999). The effect of liposomes on skin barrier structure. Skin Pharmacol Appl Skin Physiol 12, 235-246.

Coderch L, Fonollosa J, de Pera M, de la Maza A, Parra JL, Martí M, Compositions of internal lipid extract of wool and use thereof in the preparation of products for skin care and treatment, $\mathrm{N}^{\circ}$ 9901541 (1999). Spain, PCT/ES00/004244 (2000).

Coderch L, Fonollosa J, Martí M, Garde F, de la Maza A, Parra JL (2002b). Extraction and analysis of ceramides from internal wool lipids. J Am Oil Chem Soc 79, 1215-1220.

Coderch L, López O, de la Maza A, Parra JL (2003b). Ceramides and skin function. Am J Clin Dermatol 4, 107-129.

Coderch L, Soriano C, de la Maza A, Erra P, Parra JL (1995). Chromatographic characterization of internal polar lipids from wool. J Am Oil Chem Soc 72, 715-720.

de la Maza A, Manich AM, Coderch L, Bosch P, Parra JL (1995). The formation of liposomes in vitro by mixtures of lipids modelling the composition of the stratum corneum. Coll Surf A 101, 9-19.

de Pera M, Coderch L, Fonollosa J, de la Maza A, Parra JL (2000). Effect of internal wool lipid liposomes on skin repair. Skin Pharmacol Appl Skin Physiol 13, 188-195.

Esposito E, Drechsler M, Mariani P, Sivieri E, Bozzini R, Montesi L et al., (2007). Nanosystems for skin hydration: a comparative study. Int J Cosmet Sci 29, 39-47.

Fonollosa J, Campos L, Martí M, de la Maza A, Parra JL Coderch L (2004). X-ray diffraction analysis of internal wool lipids. Chem Phys Lipids 130, 159-166.

Fonollosa J, Martí M, de la Maza A, Parra JL, Coderch L (2000a). TLCFID analysis of the ceramide content of internal wool lipids. $J$ Planar Chrom 13, 119-122.

Fonollosa J, Martí M, de la Maza A, Sabés M, Parra JL, Coderch L (2000b). Thermodynamic and structural aspects of internal wool lipids. Langmuir 16, 4808-4812.

Fresta M, Puglisi G (1997). Corticosteroid dermal delivery with skinlipid liposomes. J Contr Rel 44, 141-151.

Gray GM, White RJ (1979). Epidermal lipid analysis: a novel non-phospholipid membrane system. Biochem Soc Trans 7, 1129-1131.
Kelly R, Roddick-Lanzilotta A, Vorwerk E, Coderch L (2007). Treating hair or nails with internal wool lipids. US $N^{\circ} 60 / 774,606$.

Körner A, Petrovic S, Höcker H (1995). Cell membrane lipids of wool and human hair form liposomes. Tex Res J 65, 56-58.

Lian T, Ho RJY (2001). Trends and developments in liposome drug delivery systems. J Pharm Sci 90, 667-680.

Little C, El-Sharif M, Hepher MJ (2007). The effect of solution level on calorific and dosimetric results in a 70-kHz tower-type sonochemical reactor. Ultrason Sonochem 14, 375-379.

Méndez S, Barba C, RoddickLanzilotta A, Kelly R, Parra JL, Coderch L (2008). Application of internal wool lipids to hair. Skin Res Technol 14, 448-453.

Méndez S, Martí M, Barba C, Parra JL, Coderch L (2007). Thermotropic behavior of ceramides and their isolation from wool. Langmuir $23,1359-1364$.

Pereira-Lachataignerais J, Pons R, Panizza P, Courbin L, Rouch J, López $\mathrm{O}$ (2006). Study and formation of vesicle systems with low polydispersity index by ultrasound method. Chem Phys Lipids 140, 88-97.

Ramírez R, Martí M, Manich A, Parra JL, Coderch L (2008). Ceramides extracted from wool: pilot plant solvent extraction. Tex Res $J$ 78, 73-80.

Schaefer H, Redelmeier TE (1996). Skin Barrier: Principles in Percutaneous Penetration. Basel, Karger Publishers, pp 55-58.

Schmid MH, Korting HC (1993). Liposomes for atopic dry skin: the rationale for a promising spproach. Clin Invest 71 , 649-653.

Silva R, Little C, Ferreira H, Cavaco-Paulo A (2008). Incorporation of peptides in phospholipid aggregates using ultrasound. Ultrason Sonochem 15, 1026-1032.

Teschke O, de Souza EF (2002). Liposome structure imaging by atomic force microscopy: verification of improved liposome stability during adsorption of multiple aggregated vesicles. Langmuir 18, 6513-6520.

Wartewig S, Neubert RHH (2007). Properties of ceramides and their impact on the stratum corneum structure: a review. Skin Pharmacol Physiol 20, 220-229.

Wertz PW, Abraham W, Landmann L, Downing DT (1986). Preparation of liposomes from stratum corneum lipids. $J$ Invest Dermatol 87, 582-584. 